\title{
Arterias Comunicantes del Círculo Arterial del Cerebro: Características Biométricas y Relación con el Índice Cefálico
}

\author{
Communicating Arteries of the Cerebral Arterial Circle: \\ Biometric Features and their Relation with the Cephalic Index
}

"Mandiola, E; *Alarcón, E; "Oñate, J. C.; ** del Sol, M.\& **Oave, E.

MANDIOLA, E.; ALARCón, E.; OÑATE, J. C.; DEL SOL, M. \& OLAVE, E. Arterias comunicantes del círculo arterial del cerebro: Características biométricas y relación con el índice cefálico. Int. J. Morphol., 28(3):957-961, 2010.

RESUMEN: La clínica neuroquirúrgica tiene la necesidad de un acabado conocimiento anatómico de las arterias comunicantes como integrantes del círculo arterial del cerebro, por su variabilidad morfológica, su relación compleja con las estructuras adyacentes y por la alta frecuencia observada en la conformación de aneurismas. Debido a la importancia de estos vasos, efectuamos un estudio biométrico de los mismas, relacionando datos de calibre y longitud con índice cefálico. En el sexo masculino, el calibre promedio de la ACoP fue de 1,07 mm en el lado derecho y de 1,06 mm en el izquierdo; en el sexo femenino fue de 1,12 mm en el lado derecho y de 1,14 mm en el izquierdo. En el grupo de individuos con índice braquicéfalo, el calibre de la ACoP fue de 1,15 mm y en el grupo dolicomesocéfalo fue de 1,05 mm. Su longitud fue de 18,65 mm en los braquicéfalos y de 16,44 mm en los dolicomesocéfalos.El calibre de la ACoA fue de $1,53 \mathrm{~mm}$ en el sexo masculino y de $1,05 \mathrm{~mm}$ en el femenino, mientras que su longitud fue de 2,81 mm en el sexo masculino y de 2,33 mm en el femenino.La descripción biométrica y bioantropológica muestra parámetros importantes a considerar en la clínica quirúrgica.

PALABRAS CLAVE : Anatomía; Biometría; Círculo arterial cerebral; Arterias comunicantes; Indice cefálico.

\section{INTRODUCCIÓN}

En el círculo arterial del cerebro hay una variabilidad importante en sus componentes, la cual ha sido observada en las arteriografías y técnicas de diagnóstico, como lo han mostrado los resultados obtenidos por resonancia magnética y hallazgos en el campo operatorio (González, 1959; Barbosa et al., 1969; Sengupta, 1975; Lazorthes et al., 1979; Kamath, 1981; Orlandini et al., 1985; El Khamlichi et al., 1985; Pedroza et al., 1987; Fujimoto \& Tanaka, 1989; Aydin et al.,1997; Krupa \& Krupa., 1998; Macchi et al., 2002; Karansincir et al., 2004 ). También esta variabilidad puede ser causa de la aparición de trastornos neurológicos (Hendrikse et al., 2005; Eftekhar et al., 2006; Waaijer et al., 2007; Bugnicourt et al., 2009; Martinaud et al., 2009), entre otros.

En la actualidad, los diversos procedimientos quirúrgicos en esta área, hacen necesario conocimientos precisos de anatomía aplicables a la microcirugía.

*Facultad de Medicina, Universidad Andrés Bello, Santiago, Chile.

** Facultad de Medicina, Universidad de la Frontera, Temuco, Chile.

Parcialmente financiado por Proyecto DI- UNAB- 02-05/ R
En trabajos previos hemos hecho notar, que son escasas las investigaciones que asocian características morfológicas vasculares encefálicas con caracteres bioantropológicos (Mandiola et al., 2005, 2006)

Estos factores nos han llevado a revisar y analizar las características biométricas de las arterias comunicantes anterior y posterior (ACoA y ACoP, como componentes de la estructura del círculo arterial cerebral, intentando además, asociar sus características con mediciones bioantropológicas, de las cuales obtuvimos los índices cefálicos.

\section{MATERIAL Y MÉTODO}

Se estudiaron 36 encéfalos de individuos adultos, de ambos sexos. Se analizaron las arterias comunicantes des- 
de el punto de vista biométrico, considerando su longitud y calibre.

Los datos obtenidos, se analizaron de acuerdo a las siguientes variables: lado, sexo e índice cefálico (de acuerdo a Martin \& Saller, 1957). Estos últimos se agruparon en braquicéfalos y dolicomesocéfalos (BH y DM). En la Fig. 1 se observan las dimensiones registradas en las arterias en estudio.

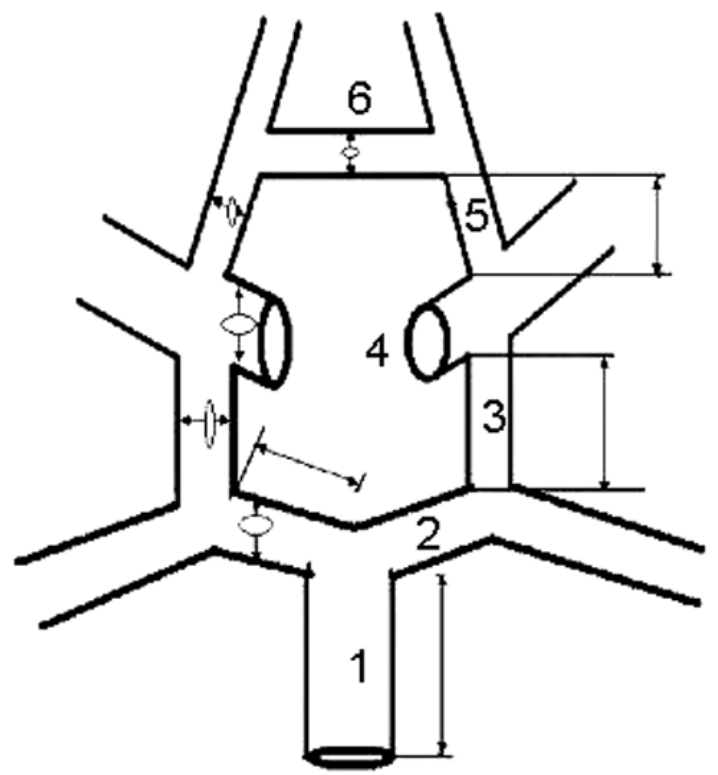

Fig 1. Dimensiones registradas en el circulo arterial del cerebro. 1. Arteria Basilar (AB). 2. Segmento pre-comunicante de Arteria Cerebral Posterior (ACP-P1). 3. Arteria comunicante posterior (ACoP). 4. Arteria Carótida Interna. 5. Segmento pre-comunicante de la arteria cerebral Anterior (ACAA1). 6. Arteria comunicante anterior.

Los resultados se analizaron estadísticamente, considerando valores promedio y desviación estándar, comparando lados, sexo e índices cefálicos a través del t-Student.

\section{RESULTADOS}

Los valores promedio del calibre y longitud de la ACoP son mostrados en la Fig. 2. Diferencias de calibre entre las arterias comunicantes posteriores se observan en la Fig. 3.

Los valores promedio del calibre de la arteria comunicante anterior son mostrados en la Fig. 4.

Las figuras 5 y 6 muestran los resultados obtenidos

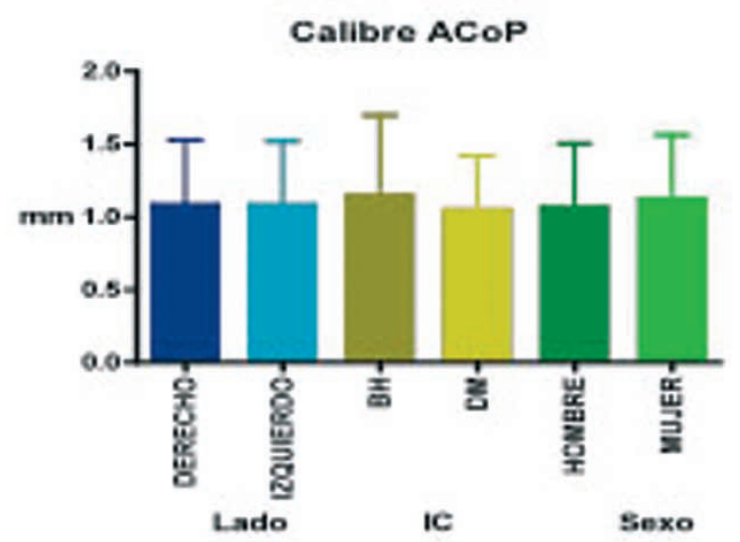

Fig. 2. Valores promedio y desviación estándar de los calibres de ACoP, según lado, índice cefálico y sexo.

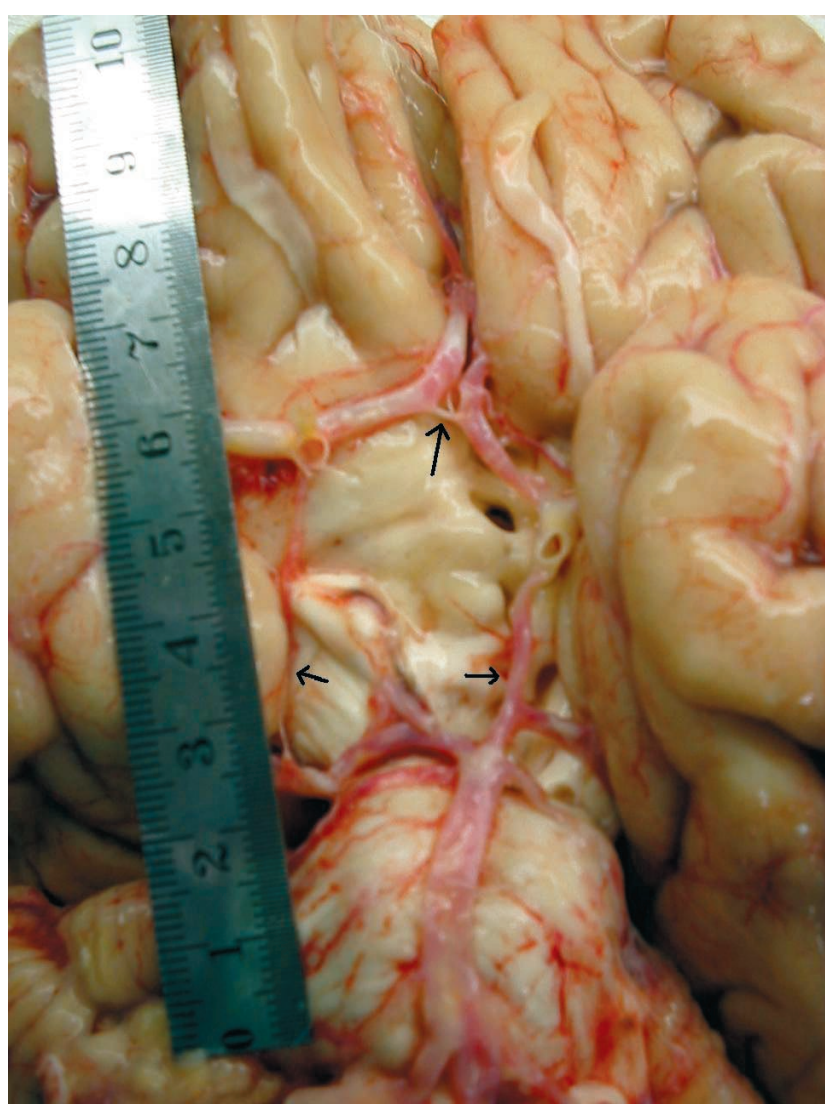

Fig 3. Visión ventral del círculo arterial cerebral. Las flechas indican arterias comunicantes anterior y posteriores.

para la variable longitud de la ACoP, según lado, índice cefálico y sexo, y, de la ACoA, según índice cefálico y sexo, respectivamente.

Considerando la presencia de una o dos arterias, observamos que en un $29 \%$ de nuestra serie la ACoA fue doble. Se observó además, la diferencia tanto en longitud como en calibre de la ACoP. 


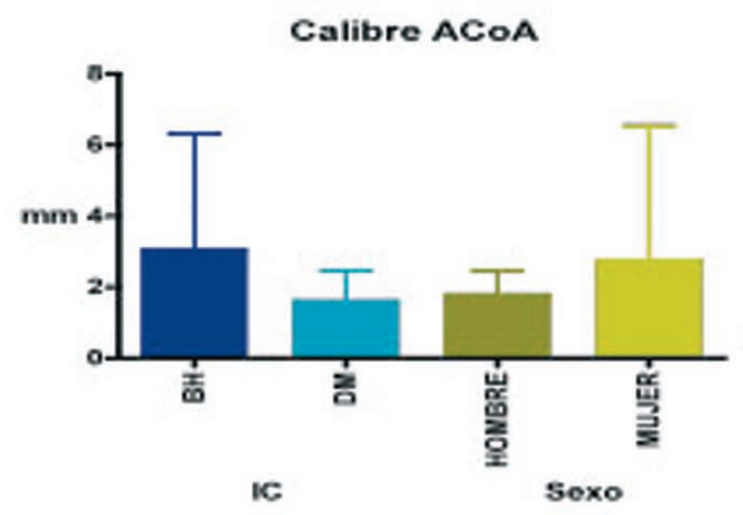

Fig. 4. Valores promedios y desviación estándar del calibre de la ACoA, según índice cefálico y sexo.

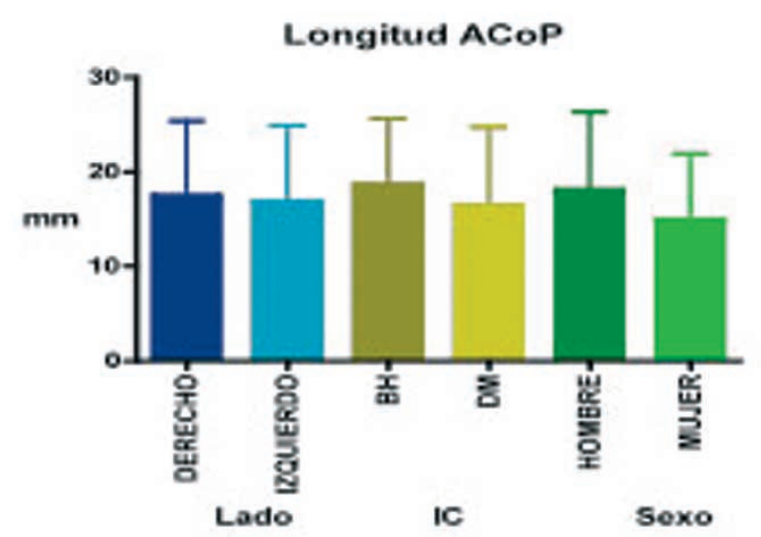

Fig. 5. Longitud promedio de la ACoP, según lado, índice cefálico y sexo.

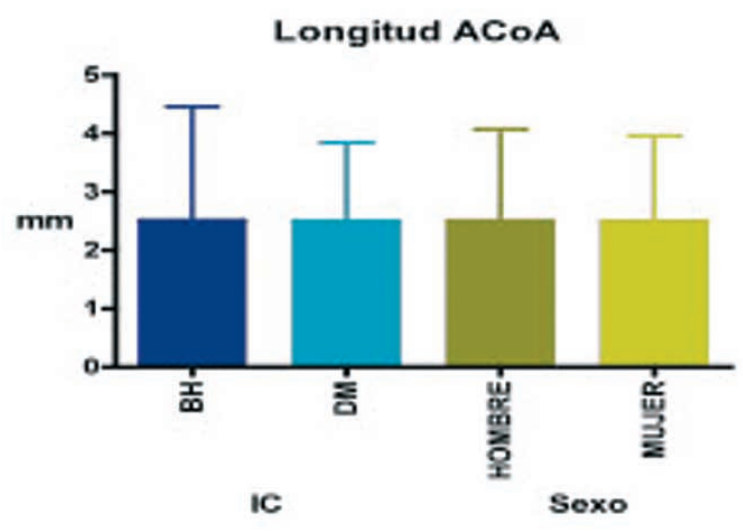

Fig. 6. Longitud promedio de la ACoA, según índice cefálico y sexo.

Al comparar estadísticamente, existe diferencia significativa $(\mathrm{p}<0.006)$ del calibre de las ACoA simples y dobles, mientras que al comparar las ACoP no hay diferencias significativas. (Fig. 7).

\section{Calibre}

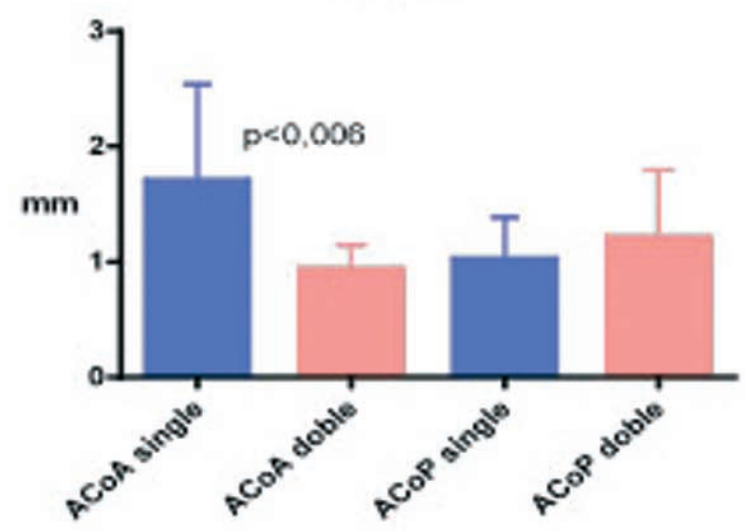

Fig. 7. Valores promedios y desviación estándar de los calibres, cuando las arterias comunicantes se presentaron únicas y dobles.

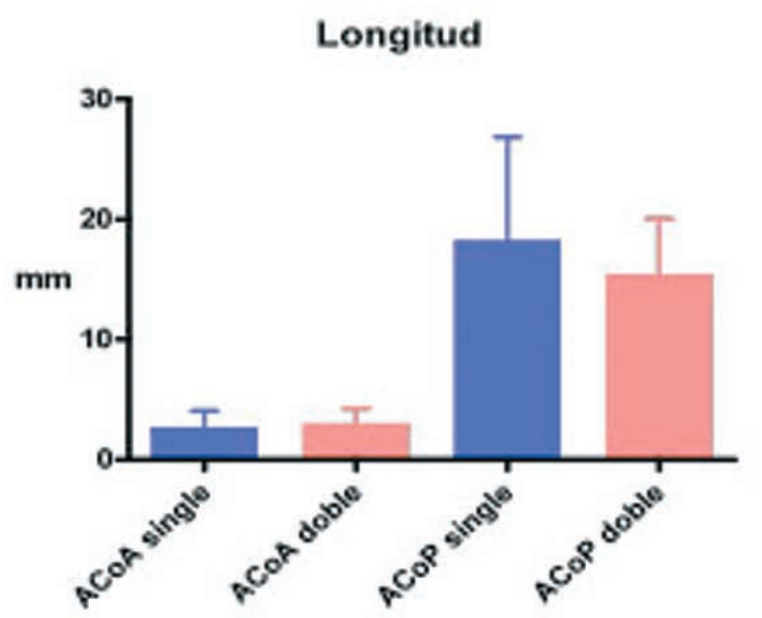

Fig. 8. Valores promedios y desviación estándar de longitud, cuando las arterias comunicantes se presentaron únicas y dobles.

En cuanto a la longitud de las ACoA dobles y singles y las ACoP, no se observaron diferencias estadísticamente significativas (Fig.8).

\section{DISCUSIÓN}

La hipoplasia o agenesia y la incidencia de aneurismas (30\%) en la ACoA reportados por Karanzincir et al. se debería a las variaciones observadas en el circulo arterial del cerebro, como ha sido mostrado en diseños y evidencias radiológicas por diversos autores (González; Pedroza et al.; Fujimoto \& Tanaka; Macchi et al.; Hendrikse et al.;Waaijer et al.; Martinaud et al.). 
La presencia de ACoA dobles (29\% de nuestros casos) aumentaría la probabilidad de aneurismas, según lo expresado por Marinkovic et al. (1989), quienes encontraron tal disposición en un $22 \%$ y Karanzincir et al. observaron un $26,6 \%$ en pacientes con esta entidad vascular. Por otra parte, es importante lo señalado por Aydin et al. donde el 60 $\%$ de los pacientes intervenidos quirúrgicamente, presentaron variaciones anatómicas en la cercanía de la ACoA.

Para Fujimoto \& Tanaka la ACoA se caracteriza por la multiplicidad de sus formas y tanto éstas como las ACoP pueden exhibir configuraciones hipoplásticas, pero que no deben confundirse con malformaciones congénitas.

El calibre de la ACoA registrado en nuestras muestras fluctuó entre 0,68 y 3,11 mm y la longitud entre 0,8 y $5,41 \mathrm{~mm}$. El $90 \%$ de las ACoA dobles tienen diámetro menor a 1,0 mm, considerándolas hipoplásticas, porcentaje diferente de lo descrito por Krupa \& Krupa, quienes encontraron en su estudio un $30 \%$ con estas características. Con valores bastante más bajos, Lazorthes et al. y El Khamlichi et al. describieron sólo un 7,5\% y $4 \%$, respectivamente.

Estos últimos autores registraron una longitud promedio para la ACoP de 13,05 mm en el lado derecho y de $12,74 \mathrm{~mm}$ en el izquierdo, resultados inferiores a los nuestros, en que registramos una longitud de $17,95 \mathrm{~mm}$ en el lado derecho, sexo masculino. En el sexo femenino, observamos una longitud de 16,50 mm en lado derecho y de 13,45 mm en el izquierdo. Resultados similares relató Kamath.
Eftekhar et al. han encontrado hipoplasia de estas arterias ( ACoP) en un $27 \%$, consideradas unilateralmente y un $33 \%$ bilateralmente, aunque para Waaijer et al. no hay diferencias significativas en la configuración entre las observaciones ipsilaterales versus contralaterales o lados derechos e izquierdos. Para Bugnicourt et al., la ausencia de las ACoP unilateral o bilateralmente, referidas como círculo arterial de la base posteriormente incompleto, lo han asociado, de acuerdo a sus hallazgos clínicos, a un factor de riesgo en el padecimiento de migraña. No hay datos de los autores mencionados que relacionen datos biométricos con bioantropológicos, como lo han señalado Mandiola et al. (2005, 2006).

En general, concordamos con lo descrito en la literatura en lo relativo a que las mediciones postmortem efectuadas no son las que se observan in vivo, aunque es posible minimizar las fuentes de error con diversas técnicas, entre otras, usando programas computacionales diseñados para ello.

El análisis detallado de caso por caso, de los diversos parámetros estudiados, permite señalar que existe variabilidad en las arterias estudiadas, conformando un círculo arterial cerebral bastante asimétrico, factores que deben ser considerados en la clínica. Es necesario un mayor número de casos para evidenciar significancia estadística más relevante y sobre todo, los aportes que se hacen considerando datos bioantropológicos.

MANDIOLA, E.; ALARCón, E.; OÑATE, J. C.; DEL SOL, M. \& OLAVE, E. Communicating arteries of the cerebral arterial circle: Biometric features and their relation with the cephalic index. Int. J. Morphol., 28(3):957-961, 2010.

RESUMEN: The neurosurgical clinic needs extensive and thorough anatomical knowledge of the communicating arteries as part of the cerebral arterial circle for their morphological variability, their complex relation as adjacent structures, and for the frequency observed in the formation of aneurisms. Considering the importance of these vessels, we conducted a biometric study of these, relating caliber and longitude information to the cephalic index. In the male, the mean caliber of the PCoA $<\mathrm{p}$ was of $1.07 \mathrm{~mm}$ on the right side and $1.06 \mathrm{~mm}$ on the left; in the female it was of $1.12 \mathrm{~mm}$ on the right side and of $1.14 \mathrm{~mm}$ on the left. In the group of individuals with brachiocephalic index, the caliber of the ACoP was of $1.15 \mathrm{~mm}$ and in the dolichomesocephalic groups was $1.05 \mathrm{~mm}$. Its longitude was $18.65 \mathrm{~mm}$ in the brachiocephalous and of $16.44 \mathrm{~mm}$ in the dolichomesochephalic. The caliber of the ACoA was of $1.53 \mathrm{~mm}$ in the male and $1.05 \mathrm{~mm}$ in the female, while its longitude was of $2.81 \mathrm{~mm}$ in the male and $2.33 \mathrm{~mm}$ in the female. The biometric and bioanthropologic description shows important parameters to be considered in clinical surgery.

KEY WORDS : Anatomy; Biometry; Cerebral arterial circle; Communicating arteries; Cephalic index.

\section{REFERENCIAS BIBLIOGRAFICAS}

Aydin, I. H.; Takei, E.; Kadioglu, H. H.;Tuzun,Y.; Kayaoglu, C. R. \& Barlas, E. Vascular variations associated with anterior communicating artery aneurysms-na intraoperative study. Minim. Invasive Neurosurg., 40(1): 17-21, 1997.
Barbosa, L.; Purriel, J. A.; Meerhoff, W. \& Medoc, J. El polígono de Willis y sus variaciones. Acta Neurol. Latinoamer., 15: 224-36, 1969.

Bugnicourt, J. M., Garcia, P. Y.; Peltier, J.; Bonnaire, B.; 
Picard, C. \& Godefroy, O. Incomplete Posterior Circle of Willis : A Risk Factor for Migraine ? Headache, 49: 879-86, 2009.

Eftekhar, B; Dadmehr, M.; Ansari, S.; Ghodsi, M.; Nazparvar, B. \& Ketabchi, E. Are the distributions of variations of circle of Willis different in different populations ? Results of an anatiomical study and review of literature. BMC Neurology, 6:22, 2006.

El Khamlichi, A.; Azouzi, M.; Bellakhdar, F.; Ouhcein, A. \& Lahlaidi, A. Configuration Anatomique du Polygone de Willis de $1^{\circ}$ adulte etudie par les Techiques d' injection. A propos de 100 cerveaux. Neurochirurgie, 31:287-93, 1985.

Fujimoto, K. \& Tanaka, O. Morphological examination of the circulus arteriosus cerebri humani (Circle of Willis). I. Anterior and posterior communicating arteries. Kaibogaku Zasshi, 64 (5) :481-9, 1989.

González, A. A. Circulo arterial de Willis. Sus medidas externas y variaciones anatómicas. Acta Neurol. Latinoamer., 5:1-6, 1959.

Hendrikse, J.; Raamt, v. F.; Graaf v. der Y.; Mali,W. \& Grond v.der, J. Distribution of Cerebral Blood Flow in the Circle of Willis. Radiology, 235:184-9, 2005.

Kamath, S. Observations en the length and diameter of vessels forming the circle of Willis. J. Anat., 133(3):419-23, 1981.

Karansincir, S.; Ada, E.; Sarilma, Z. A.; Yalcin, O.; Vidinl, I. B. \& Sabin, E. Frequency of vascular variations and anomalies accompanying intracranial aneurysms. Tani Girisim Radiol., 10(2):103-9, 2004.

Krupa, B. \& Krupa, U. Variations of the anterior communicating artery. Folia Morphol., 57(3):233-40, 1998.

Lazorthes, G.: Gouaze, A.; Santini, J-J. \& Salamon, G. Le circle arteriel du cerveau (circulus arteriosus cerebri). Anat. Clinica, 1: 241-57, 1979.

Mandiola, E.; Alarcón, E.; Del Sol, M.; Olave, E.; Montero, C.; Sanhueza, P.; Oñate, J. C. Análisis biométrico de las arterias comunicante anterior y cerebral anterior en el Segmento Precomunicante del círculo arterial cerebral. Int. J. Morphol., 23(2): 171$6,2005$.
Mandiola, E.; Alarcón, E.; Oñate, J. C.; Sanhueza, P.; Del Sol, M.; Olave, E. Biometría de las arterias comunicantes posteriores y cerebrales posteriores em su segmento precomunicante (P1) en el círculo arterial del cerebro (Willis). Int. J. Morphol., 24(4):601-6, 2006.

Macchi, C.; Lova, R. M.; Miniati, B.; Gulisano, M.; Pratesi, C.; Conti, A. A. \& Gensini, G. F. The circle of Willis in healthy older persons. J. Cardiovasc Surg., 43(6):88790, 2002.

Marinkovic', S.; Kovacevic', M. \& Millisavljevic', M. Hypplasia of the proximal segment of the anterior cerebral artery. Anat Anz., 168:45-154, 1989.

Martin, R.\& Saller, K. Lehrbuch der Anthropologie. Stuttgart, Gustav Fischer Velgar, 1957. V.1. pp. 317-79.

Martinaud, O.; Perin, B.; Gerardin, E.; Proust, F.; Bioux,S.; Le Gars, D.; Hannequin, D. \& Godefroy, O. Anatomy of executive deficit following ruptured anterior communicating artery aneurysm. European J. Neurology, 16:595-601, 2009.

Orlandini, G.; Ruggiero, C.; Oralandini, S. Z.; Gulisano, M. Blood vessel size of circulus arteriosus cerebri (circle of Willis): A Statistical Research on 100 Human Subjects. Acta Anat., 123:72-6, 1985.

Pedroza, A.; Duvojny, M.; Cabezudo, J.; Umansky, F.; Berman, K.; Diaz, F.; Ausman, J. \& Mirchardani, H.G. Microanatomy of the posterior communicating artery. Neurosurg., 20 (2):228-35, 1987.

Sengupta, R. P. Anatomical variations in the origin of the posterior cerebral artery demostrated by carotid angiography and their significance in the direct surgical treatment of posterior communicating aneurysms. Neurochirurgia, 18(2):33-42, 1975.

Waaijer, A.; Leeuwen v. M. S.; Worp v. der H. B.; Verhagen, H. J. M.; Mali, W. P. T. M. \& Velthuis, B. K. Cerebrovasc Dis., 23: 267-74, 2007.

Dirección para correspondencia:

Prof. Dr. Eduardo Mandiola

Facultad de Medicina

Departamento de Morfología

Universidad Andrés Bello

República 275, Piso 2

Santiago

CHILE

E-mail: emandiola@unab.cl
Recibido : 14-03-2010

Aceptado : 27-06-2010 
